\title{
UTILIZAÇÃO DA TÉCNICA DE TERMOLUMINESCÊNCIA PARA CARACTERIZAÇÃO DE UMA NOVA MATRIZ VÍTREA
}

\author{
P. Z. Ferreira*, G. S. M. Carvalho*, D. M. Cunha*, N. O. Dantas*, A. C. A. Silva*, \\ L. P. Neves***, L.V. E. Caldas $* *$, B.N. S. Carrera***, S. Watanabe***, A. P. Perini* \\ * Instituto de Física, Universidade Federal de Uberlândia (INFIS/UFU), Caixa Postal 593, 38400- \\ 902, Uberlândia, MG, Brasil \\ ** Instituto de Pesquisas Energéticas e Nucleares - Comissão Nacional de Energia Nuclear \\ (IPEN/CNEN-SP), 05508-000, São Paulo, SP, Brasil \\ *** Instituto de Física da Universidade de São Paulo (IFUSP), 05508-090, São Paulo, SP, Brasil \\ e-mail:pamela_zafe@hotmail.com
}

\begin{abstract}
Resumo: A utilização de dosímetros é muito importante na monitoração de radiação ionizante, que pode ser empregada na área médica, industrial e ambiental. A técnica de termoluminescência é muito utilizada em dosimetria de doses altas, que inclui atividades como gamagrafia, esterilização de materiais cirúrgicos, irradiação de alimentos, etc. As principais vantagens de materiais dosimétricos termoluminescentes são as suas pequenas dimensões, o fato de não necessitarem de cabos ou equipamentos auxiliares durante a medida da dose e suas altas sensibilidades para detecção de radiação. A técnica termoluminescente consiste basicamente na detecção de um sinal luminescente, de um material previamente irradiado, por meio de um estímulo térmico. Neste trabalho, a técnica de termoluminescência foi utilizada para estudar uma nova matriz vítrea, com composição nominal

$20 \mathrm{Li}_{2} \mathrm{CO}_{3} .10 \mathrm{Al}_{2} \mathrm{O}_{3} .10 \mathrm{BaO} .60 \mathrm{~B}_{2} \mathrm{O}_{3}(\mathrm{~mol} \%)$ irradiada com doses entre 10 Gy e 900 Gy em uma fonte de ${ }^{60} \mathrm{Co}$. Os resultados obtidos mostraram que esta nova matriz vítrea apresenta uso potencial em dosimetria de altas doses.
\end{abstract}

Palavras-chave: dosimetria, termoluminescência, novos materiais.

\footnotetext{
Abstract: The use of dosimeters is very important in ionizing radiation monitoring, which can be used in medical, industrial and environmental field. Thermoluminescence technique is widely used for highdose dosimetry, which includes activities such as gammagraphy, sterilization of surgical instruments, food irradiation, among other activities. The main advantages of thermoluminescent dosimetric materials are their small size, the fact that they do not require cables and secondary equipment during measurement of dose and their high sensitivity for radiation detection. The thermoluminescent technique consists basically in the detection of a luminescent signal from a previously irradiated material using thermal stimulus. In this work, the thermoluminescence technique was used to study a new glass matrix, with nominal composition $20 \mathrm{Li}_{2} \mathrm{CO}_{3} .10 \mathrm{Al}_{2} \mathrm{O}_{3} .10 \mathrm{BaO} .60 \mathrm{~B}_{2} \mathrm{O}_{3}$ (mol\%) irradiated with doses between $10 \mathrm{~Gy}$ and $900 \mathrm{~Gy}$ using a ${ }^{60} \mathrm{Co}$
}

source. The results showed that this new glass matrix has potential use in high-dose dosimetry.

Keywords: dosimetry, thermoluminescence, new materials.

\section{Introdução}

Termoluminescência é o fenômeno caracterizado pela emissão de luz de um material aquecido, sendo este sido previamente irradiado. A quantidade de luz emitida é proporcional à dose de radiação absorvida pelo material. O fenômeno de termoluminescência já é conhecido há muito tempo desde que Robert Boyle, em 1663, observou no escuro a emissão de luz através de um diamante aquecido pela sua temperatura corporal [1]. A utilização da termoluminescência como uma técnica em dosimetria da radiação foi sugerida por Farrington Daniels e seu grupo de pesquisa [1].

Alguns materiais emitem luz quando aquecidos e são denominados materiais termoluminescentes (TL). Algumas características são importantes num material TL, tais como: alta sensibilidade, resposta linear em função da dose absorvida, intensidade da luz emitida e temperatura de emissão [2]. Existem diversos materiais que podem ser aplicados em termoluminescência, tanto naturais quanto artificiais. Dentre os naturais podemos citar o $\mathrm{BeO}, \mathrm{o}_{2} \mathrm{Al}_{2} \mathrm{O}_{3}$ e o $\mathrm{CaF}_{2}$ (fluorita), já entre os artificiais os mais utilizados são: $\mathrm{LiF}: \mathrm{Mg}, \mathrm{Ti}$; $\mathrm{LiF}: \mathrm{Mg}, \mathrm{P}, \mathrm{Cu} ; \quad \mathrm{LiF}: \mathrm{Dy} ; \quad \mathrm{CaSO}_{4}: \mathrm{Dy} ; \quad \mathrm{Li}_{2} \mathrm{~B}_{4} \mathrm{O}_{7}: \mathrm{Mn}$; $\mathrm{Li}_{2} \mathrm{~B}_{4} \mathrm{O}_{7}: \mathrm{Cu}[3 ; 4]$.

Diversas pesquisas estão sendo feitas para melhorar as características dos materiais $\mathrm{T} \mathrm{L}$ por meio de sua preparação, utilizando diferentes métodos de síntese ou pela dopagem com diferentes impurezas $[5 ; 6]$. Alguns vidros também apresentam potencial para serem aplicados como dosímetros. Uma das vantagens de se utilizar o vidro como detector de radiação é que este apresenta dimensão reduzida, podendo ser manipulado facilmente e têm baixo custo de produção [7]. Existem trabalhos recentes que mostram o uso potencial de materiais vítreos para dosimetria das radiações $[8 ; 9]$.

A técnica de termoluminescência pode ser aplicada para dosimetria ambiental, pessoal e de doses altas. A 
dosimetria de doses altas, que envolve doses num intervalo de 10 Gy a $100 \mathrm{kGy}$, está se tornando cada vez mais comum, sendo aplicada em atividades tais como: gamagrafia, esterilização de materiais cirúrgicos, irradiação de alimentos, cura de tintas e vernizes, irradiação de pedras semipreciosas, polimerização de cabos e em atividades com reatores nucleares, entre outras atividades [10].

Notando o fato de que atividades que envolvem radiação de doses altas estão se tornando cada vez mais comuns, surge, então, a necessidade de controle da radiação envolvida nestas práticas. Sendo assim, este trabalho pretende caracterizar uma nova matriz vítrea, com composição nominal de $20 \mathrm{Li}_{2} \mathrm{CO}_{3} .10 \mathrm{Al}_{2} \mathrm{O}_{3} .10 \mathrm{BaO} \cdot 60 \mathrm{~B}_{2} \mathrm{O}_{3}(\mathrm{~mol} \%)$, avaliada para diferentes doses: 10, 50, 100, 200, 500, 700 e 900 Gy, utilizando-se a técnica de termoluminescência.

\section{Materiais e métodos}

Síntese do sistema vítreo - A síntese do material vítreo foi realizada pelo método de fusão tradicional, em forno de carbeto de silício, a uma temperatura de $1340^{\circ} \mathrm{C}$ por 15 minutos sob atmosfera de carbono. A amostra foi resfriada utilizando-se duas chapas de bronze-latão a $0^{\circ} \mathrm{C}$ e tratada termicamente a $350^{\circ} \mathrm{C}$ por 12 horas. $\mathrm{O}$ material vítreo obtido é mostrado na Figura 1.

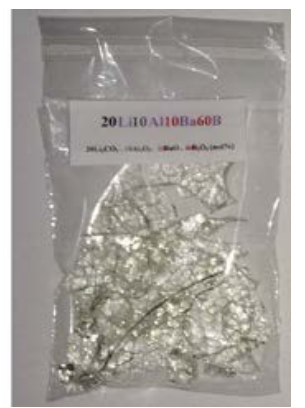

Figura 1: Sistema vítreo, com composição nominal de $20 \mathrm{Li}_{2} \mathrm{CO}_{3} .10 \mathrm{Al}_{2} \mathrm{O}_{3} .10 \mathrm{BaO} .60 \mathrm{~B}_{2} \mathrm{O}_{3}(\mathrm{~mol} \%)$, testado neste trabalho para dosimetria de doses altas.

Irradiação - As amostras foram irradiadas utilizan-

do-se uma fonte de ${ }^{60} \mathrm{Co}$, sistema Gamma-Cell 220 fabricado pela Atomic Energy of Canada, à temperatura ambiente, com uma taxa de dose de 1,38 kGy/h. Este sistema encontra-se localizado no Centro de Tecnologia das Radiações (CTR) do Instituto de Pesquisas Energéticas e Nucleares. Placas de Lucite com espessura de 3,0 $\mathrm{mm}$ foram utilizadas de modo a manter o equilíbrio eletrônico das amostras durante as irradiações.

Medições de Termoluminescência - As medições termoluminescentes foram realizadas da temperatura ambiente até $400^{\circ} \mathrm{C}$, utilizando um fluxo constante de $\mathrm{N}_{2}$ de 2,5 L/min e uma taxa de aquecimento de $10^{\circ} \mathrm{C} / \mathrm{min}$. Para realização das medidas, foi empregado um sistema leitor Harshaw TLD modelo 4500 e um computador pessoal para a aquisição dos dados (Figura 2).

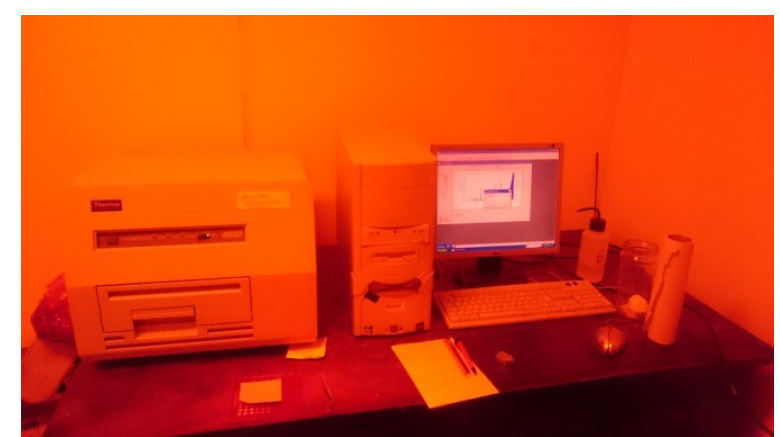

Figura 2: Sistema leitor Harshaw TLD empregado para obtenção das medidas termoluminescentes.

\section{Resultados}

De forma a caracterizar esta nova matriz vítrea, foram obtidas as curvas de emissão termoluminescente e a curva de dose-resposta.

As doses utilizadas para irradiação da matriz vítrea foram de 10, 50, 100, 200, 500, 700 e 900 Gy. Para cada dose, foi obtida uma curva de emissão TL, que são apresentadas na Figura 3. Durante as medições foi utilizada a área integrada entre $50^{\circ} \mathrm{C}$ e $400^{\circ} \mathrm{C}$.

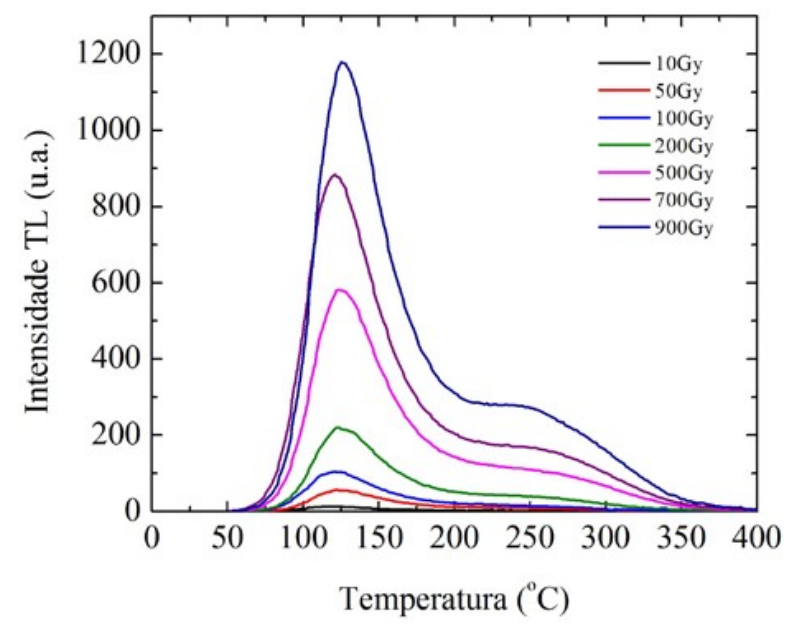

Figura 3: Curvas de emissão TL da matriz vítrea com composição nominal

de

20Li CO .10Al O $\mathrm{O}_{2} .10 \mathrm{BaO} .60 \mathrm{~B} \mathrm{O}_{3}(\mathrm{~mol} \%)$ para doses altas.

A curva de dose-resposta é representada pela resposta termoluminescente do material em função da dose de radiação absorvida por este material. Para o levantamento da curva de dose-resposta a matriz vítrea foi irradiada com as doses de 10 Gy a 900 Gy. Para cada valor de dose foram realizadas cinco medições de forma a determinar o respectivo desvio padrão para cada dose.

A curva de dose-resposta obtida para esta matriz vítrea é mostrada na Figura 4. 


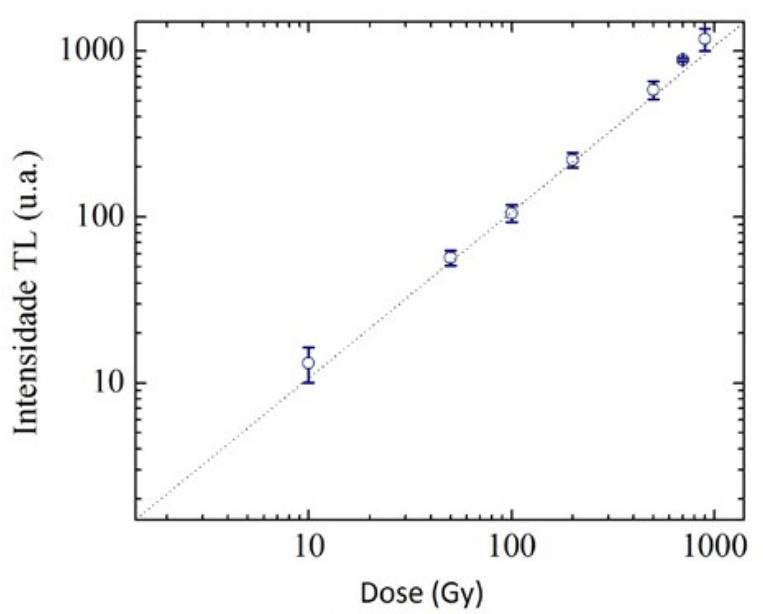

Figura 4: Curva de dose-resposta da matriz vítrea com composição nominal de $20 \mathrm{Li}_{2} \mathrm{CO}_{3} .10 \mathrm{Al}_{2} \mathrm{O}_{3} .10 \mathrm{BaO} .60 \mathrm{~B}_{2} \mathrm{O}_{3} \quad(\mathrm{~mol} \%)$, com os

respectivos desvios padrões.

\section{Discussão}

Pode-se observar que a intensidade termoluminescente na Figura 3 cresce com a dose absorvida. As doses de 10, 50, 100 e 200 Gy apresentam intensidade do sinal termoluminescente muito baixa e os picos não tão bem definidos quando comparados com as doses de 500, 700 e 900 Gy que apresentam picos bem definidos em $125^{\circ} \mathrm{C}$, além de uma intensidade do sinal TL muito alta.

Para as doses avaliadas neste trabalho, a curva de dose-resposta, mostrada na Figura 4, apresenta um comportamento linear, sendo esta uma importante propriedade para um dosímetro termoluminescente, já que materiais que apresentam comportamento nãolinear devem passar por cuidadosa calibração e correção para sua utilização.

A curva dose-resposta está relacionada a processos de transferência de carga tanto durante a irradiação, quanto durante o aquecimento, podendo revelar a existência de processos de competição entre os defeitos pela captura de portadores de carga. A sublinearidade ou supralinearidade pode causar erros na estimativa da dose no dosímetro TL, porém não exclui o uso deste dosímetro, mas requer a calibração cuidadosa e correção para evitar erros adicionais. Sendo assim, o comportamento linear apresentado na curva de doseresposta permite que o material, caracterizado neste trabalho, possa ser utilizado para avaliação da dose de radiação sem calibração. Portanto, a matriz vítrea analisada pode ser aplicada para dosimetria de doses altas no intervalo de dose avaliado.

\section{Conclusão}

Neste trabalho, uma nova matriz vítrea foi estudada e analisada para uso em dosimetria de doses altas. A matriz vítrea foi irradiada com diferentes doses: 10, 50, 100, 200, 500, 700 e 900 Gy. Pela análise do comportamento das curvas de emissão e dose-resposta, pode-se concluir que a matriz vítrea avaliada neste trabalho apresenta uso potencial como um detector em dosimetria de doses altas, relacionado a atividades como gamagrafia, cura de tintas e vernizes, esterilização de materiais cirúrgicos, irradiação de pedras semipreciosas, entre outras atividades. Portanto, este trabalho trouxe como contribuição científica o estudo de um novo material dosimétrico para ser aplicado em medições de radiação ionizante. Como trabalhos futuros, novos testes de caracterização (reprodutibilidade e perda de sinal), deste material, serão propostos, para então, passar a utilizá-lo em dosimetria de doses altas.

\section{Agradecimentos}

Este trabalho recebeu apoio financeiro das seguintes agências: Fundação de Amparo à Pesquisa do Estado de Minas Gerais (FAPEMIG, Projeto n ${ }^{0}$. APQ-02934-15), CAPES (Projeto Pro-Estratégia no. 1999/2012), CNPq (Projeto no. 157593/2015-0) e INCT (Projeto INCT Metrologia das Radiações em Medicina).

\section{Referências}

[1] Mckeever S, Moscovitch M, Townsend P. Thermoluminescence Dosimetry Materials: Properties and Uses. England: Nuclear Technology Publishing; 1995. p. 204.

[2] D’Amorim RA. Caracterização de detectores de radiação de espodumênio [tese de doutorado]. Aracajú: Universidade Federal de Sergipe; 2012.

[3] Barthe J, Portal G. Technologie des lecteurs de dosimètres thermoluminescents. Radioprotection. 1990; 25(02):135-56.

[4] Campos LL. Termoluminescência de materiais e sua aplicação em dosimetria da radiação. Cerâmica. São Paulo. 1998; 44(290):244-51.

[5] Horowitz Y, Olko P. The effects of ionization density on the thermoluminescence response (efficiency) of LiF:Mg, Ti and LiF:Mg,Cu,P. Radiation Protection Dosimetry. 2004; 109(4):331-48.

[6] Zahedifar M, Sadeghi E. Thermoluminescence dosimetry properties of new $\mathrm{Cu}$ doped $\mathrm{CaF}_{2}$ nanoparticles. Radiation Protection Dosimetry. 2013; 157(3):303-9.

[7] Teixeira MI. Propriedades dosimétricas de vidros comerciais e de areia para doses altas [tese de doutorado]. São Paulo: Universidade de São Paulo; 2004.

[8] Laopaiboon R, Bootjomchai C. Thermoluminescence studies on alkali-silicate glass doped with dysprosium oxide for use in radiation dosimetry measurement. Journal of Luminescence. 2015; 158: 275-280.

[9] Mhareb MH, Hashim S, Ghoshal SK, Alajerami YS, Saleh MA, Razak NA, Azizan AS. Thermoluminescence properties of lithium magnesium borate glasses system doped with dysprosium oxide. Luminescence. 2015; 30(8): 1330-1335. 
[10] Vila GB. Caracterização de silicatos e carbonatos de cálcio aplicados à dosimetria de doses altas [tese de doutorado]. São Paulo: Universidade de São Paulo; 2012. 